\section{Removal of template DNA from cRNA preparations by combined oligo(dT) affinity chromatography and DNase I digestion}

\author{
Markus Stöcher and Jörg Berg \\ General Hospital Linz, A-4020 Linz, Austria
}

BioTechniques 36:480-482 (March 2004)

Reverse transcription PCR (RTPCR) is a very sensitive tool for the detection and quantification of RNA, including mRNA, and is widely used in research and in diagnostic applications $(1,2)$. RT-PCR assays include RNA controls that are co-analyzed in parallel or within the same reaction tube as internal controls or as quantification standards (1). As an alternative to using mRNA transcribed from housekeeping genes, RNA controls can be conveniently prepared as complementary RNA (cRNA) from template DNA by using in vitro transcription (IVT). Prior to the use of cRNA as controls or quantification standards in RT-PCR assays, template DNA must be removed from the prepared cRNA in order to obtain accurate conditions of control or accurate quantification of results (3).

DNA templates can be removed from cRNA preparations by various methods, such as selective DNA extraction, gel fractionation, selective RNA purification, and DNase I digestion, which is probably the most widely used method $(1,3)$. After DNA template removal, cRNA preparations should be analyzed for remnants of template DNA by, for example, PCR without the prior reverse transcription step. In a recent study, such PCR analyses have been carried out as "no reverse transcription controls" by using cRNA preparations that had been subjected to a spectrum of different DNA removal procedures. The PCR analyses showed that all the various DNA removal procedures yielded cRNA preparations that consistently contained remnants of template DNA to varying extents (3).

To address this issue, we report a procedure that is able to remove template DNA from cRNA preparations beyond detection by real-time PCR on the LightCycler ${ }^{\circledR}$ instrument (Roche Applied Science, Mannheim, Germany). The procedure involves a negative and a positive selection principle and is based on a commercially available mRNA purification kit. As a prerequisite for this approach, the cRNA must contain a poly(A) tail. After IVT, the synthesized cRNA is selectively removed from template DNA by oligo(dT) affinity chromatography, and the remaining template DNA is degraded by DNase I treatment.

The procedure was developed in preparing an internal cRNA control for an enterovirus-specific RT-PCR assay. The assay-specific IVT template DNA was generated by three consecutive preparative PCRs using composite primers (Table 1$)$ as described previously $(4,5)$. The IVT template consists of a stretch of the bacterial neomycin phosphotransferase gene (neo) $\left(\mathrm{GenBank}^{\circledR}\right.$ accession no. M28248, bp 2407-2521), which is bordered by enterovirus-specific sequences. Thus, the first primer set amplified the respective stretch of neo from the plasmid pGLE-neo (5) and added enterovirus-specific sequences to both the $5^{\prime}$ and $3^{\prime}$ ends. The second primer set extended the enterovirus-specific sequences to both the $5^{\prime}$ and $3^{\prime}$ ends (GeneBank accession no. M74567, bp 453-492 and bp 582-629), and the third primer set added a $\mathrm{T} 7$ promoter core sequence at the $5^{\prime}$ end and the poly(A) sequence at the $3^{\prime}$ end of the construct. Thus, IVT and separation of the cRNA from template DNA by oligo(dT) affinity chromatography were enabled.

The DNA construct was transcribed in vitro by using the T7-MEGAshortscript $^{\mathrm{TM}}$ kit (Ambion, Austin, TX, USA) according to the manufacturer's instruction. Template DNA (250 ng) was transcribed in a $20-\mu \mathrm{L}$ transcription reaction for $4 \mathrm{~h}$ at $37^{\circ} \mathrm{C}$. Thereafter, the obtained cRNA was separated from template DNA by using a commercially available mRNA purification kit, the MagNA Pure ${ }^{\mathrm{TM}}$ LC mRNA HS Kit (Roche Applied Science). This kit uses biotin-labeled oligo(dT) in conjunction with streptavidin-coated magnetic particles to isolate poly(A)-containing RNA from cell lysates. Also, the kit includes a DNase I digestion step to degrade any remaining DNA (Instruction Manual, Version 1, January 2003; http://www.roche-applied-science.com/ pack-insert/3267393a.pdf). The $20 \mu \mathrm{L}$ IVT mixture was diluted with PCRgrade water to yield $1200 \mu \mathrm{L}$ and was then subjected to automated poly(A) RNA purification on the MagNA Pure LC instrument (Roche Applied Science) according to the manufacturer's instructions. The poly(A)-containing cRNA was eluted with $50 \mu \mathrm{L}$ elution buffer.

The purified cRNA preparations were analyzed for any remaining template DNA by real-time PCR on the LightCycler instrument without reverse transcription by using the respective target specific primers (see Table 1, the underlined sequences of the 2 nd primer set) (6) and the neo-specific fluorescence resonance energy transfer (FRET) hybridization probes (TIB MOLBIOL, Berlin, Germany) (Table 1) as described previously (5). Briefly, FastStart ${ }^{\mathrm{TM}}$ DNA Master Hybridization Probes (Roche Applied Science) were supplemented with $4 \mathrm{mM} \mathrm{MgCl}$ (final concentration), 0.2 $\mu \mathrm{M}$ forward and reverse primers each, and $0.2 \mu \mathrm{M}$ neo-specific FRET-hybridization probes each. PCR was carried out in a $20-\mu \mathrm{L}$ volume $(15 \mu \mathrm{L}$ reaction mixture and $5 \mu \mathrm{L}$ cRNA preparation) at $95^{\circ} \mathrm{C}$ for 9 min followed by 45 cycles of $2 \mathrm{~s}$ at $95^{\circ} \mathrm{C}, 10 \mathrm{~s}$ at $60^{\circ} \mathrm{C}$, and $15 \mathrm{~s}$ at $72^{\circ} \mathrm{C}$. The products were analyzed by melting curve analysis by applying $95^{\circ} \mathrm{C}$ for $1 \mathrm{~min}, 60^{\circ} \mathrm{C}$ for $1 \mathrm{~min}$, and $50^{\circ} \mathrm{C}$ for $2 \mathrm{~s}$, followed by increasing the temperature from $50^{\circ}$ to $85^{\circ} \mathrm{C}\left(0.2^{\circ} \mathrm{C} / \mathrm{s}\right)$ and continuous fluorescence recording. Real-time PCR analysis of the purified cRNA did not lead to any amplification product from four purifications analyzed in quadruplicate [Figure 1A, oligo(dT)purified plus DNase I-treated cRNA]. Real-time PCR analysis of cRNA preparation without the template removal 
Table 1. Composite Primers Used for the Generation of the Template DNA and Primers and Probes Used for the Enterovirus-Specific Real-Time PCR

1st PCR
Forward: 5'-TAA CTG CGG AGC ACA TAC CCC ATC ATG GCT GAT GCA ATG-3'
Reverse: 5'-ATT GTC ACC ATA AGC AGC CAA AGA CCG GCT TCC ATC CGA-3'
2nd PCR
Forward: 5'-CCC TGA ATG CGG CTA ATC CTA ACT GCG GAG CAC ATA CCC-3'
Reverse: 5'-CAA TAA CTA TAT GGT AAC AAC CTG TCA ATT GTC ACC ATA AGC AGC CA-3'
3rd PCR
Forward: 5'-GCG TAA TAC GAC TCA CTA TAG GGA GAG GAG CCC TGA ATG CGG CTA AT-3'
Reverse: 5'-TTT TTT TTT TTT TTT TTT TTT CAA TAA CTA TAT GGT AAC AAC CTG-3'
Probes
NEO-FLU: 5'-GCT GCA TAC GCT TGA TCC GGC T-Fluorescein-3'
NEO-LCR: 5'-LCR 705-CCT GCC CAT TCG ACC ACC AAG C-Phosphor-3'
aUnderlined sequences represent the primers used for the enterovirus-specific real-time PCR.

procedure yielded amplification signals after just four cycles of PCR (Figure 1A, nonpurified cRNA). When nonpurified cRNA preparations were serially diluted in log-fold fashion, PCR products were obtained up to a dilution of $1: 10^{10}$ (data not shown). When cRNA preparations were treated either with DNase I only or with oligo(dT) affinity chromatography only, amplification signals were obtained after approximately 25 to 28 cycles, respectively (Figure 1A). The experiments demonstrate that only the combined procedure of oligo(dT) affinity chromatography and DNase I digestion eliminated template DNA in cRNA preparations beyond detection of real-time PCR on the LightCycler instrument.

In order to test whether the applied DNA removal procedure yielded analyzable cRNA, purified cRNA [oligo(dT)purified plus DNase I-treated cRNA] preparations were serially diluted from $1: 10^{5}$ to $1: 10^{10}$ in log-fold steps and analyzed by reversre transcription followed by real-time PCR on the LightCycler instrument. Reverse transcription was carried out by using SuperScript ${ }^{\mathrm{TM}}$ III (Invitrogen, Carlsbad, CA, USA) according to the manufacturer's instruction. Two microliters of purified cRNA were reverse-transcribed in $20-\mu \mathrm{L}$ reactions for $1 \mathrm{~h}$ at $55^{\circ} \mathrm{C}$ using $0.2 \mu \mathrm{M}$ of reverse primer (Table 1) (6). Figure 1B shows that the obtained cRNA was analyzable by real-time RT-PCR up to a dilution of $1: 10^{10}$. However, the purified cRNA dilution of 1: $10^{10}$ yielded inconsistent positive and negative results, with two positive amplifications out of four replicate experiments (one positive result is shown in Figure 1B). When the PCR products were also analyzed by agarose gel electrophoresis, the expected size of $173 \mathrm{bp}$ for the cRNA derived control was obtained in all real-time PCR positive reactions (data not shown).

The results of the RT-PCR analyses suggest that the applied purification procedure did not negatively affect the amplification capacity of the obtained purified cRNA. As shown in Figure $1 \mathrm{~B}$, the purified cRNA preparations can be diluted up to at least $1: 10^{9}$ to yield consistent amplification products in real-time PCR. In the respective diagnostic RT-PCR assay, the purified cRNA could be used at a dilution of $1: 10^{9}$ as an internal control. Theoretically, this means that the amount of purified cRNA from a single IVT could suffice for about $10^{10}$ RT-PCRs. When quantification is desired, the obtained purified cRNA must be quantified prior to use. Then, suitable dilutions thereof will range between $1: 10^{5}$ and $1: 10^{8}$.

The issue of whether the purified cRNA is completely free of template DNA is not resolved by the methodology that we have used, as the absence of PCR amplification in the purified cRNA preparations must not necessarily be interpreted as a total absence of template DNA. Rather, the analytical sensitivity of real-time PCR on the LightCycler instrument has been shown to be as low as a single copy of DNA per capillary (7). This sensitivity may not always be obtained, as many diagnostic real-time PCR assays on the LightCycler instrument showed a sensitivity of approximately 5 to 10 DNA copies per capillary (8). Since the nonpurified cRNA preparations showed a DNA titer of
$1: 10^{10}$ in the real-time PCR analysis, the presented procedure should decrease template DNA by a factor of $10^{10}$ at least. We have not obtained any amplification of template DNA from four cRNA purifications analyzed in quadruplicate. We estimate, therefore, that in the worst case, our purified cRNA preparations will theoretically contain less than 50-100 copies of template DNA. Considering that the purified cRNA is used in dilutions of $1: 10^{5}$ or much lower, we suspect that any analytical error will be markedly less than $1 \%$. As the coefficients of variance of realtime PCR on the LightCycler instrument are about $2 \%-5 \%(7,8)$, any overestimation of copy numbers using dilutions of the purified cRNA should be lower than the coefficients of variance of the respective real-time PCR assay.

This procedure, which removes template DNA from cRNA preparations and is performed in an automated fashion, was found to be reproducible and standardized. The generation of the template DNA by the composite primer technology was rapid and convenient. Other procedures to generate template DNA, such as the cloning of internal control constructs, should also be feasible with this DNA removal procedure provided that the constructs contain a poly(A) tail. As instruments for automated nucleic acid purification become increasingly available in diagnostic and analytical laboratories, the method described here should also be applicable for use with other instruments (i.e., automated nucleic acid purification in conjunction with an mRNA purification method). In addition, this automated procedure ought to be applicable to a manual work up with the assistance of a magnet, since all of the steps and the volumes of the procedure are described in detail in the instruction manual of the kit. In general, any mRNA isolation method based on oligo(dT) affinity chromatography [e.g., Oligotex $^{\circledR}$ (Qiagen, Valencia, CA, USA)] combined with a DNase I digestion step should be applicable and should yield similar results. The manufacturer did not, upon request, disclose the concentrations of the components of the kit that we used. As the kit was designed to isolate mRNA from up to $10^{7}$ peripheral blood leucocytes, we believe that both oligo(dT) and 
DNase I are used in great excess. Importantly, the DNase I was completely free of RNases, as outlined in the manufacturer's instructions.

The cRNA synthesized by IVT should be purified from the IVT reagents prior to use as internal controls or standards in the RT-PCR assays. Since the mRNA isolation kit used in this study is designed to also separate proteins from the mRNA, our procedure meets this necessity, too. Apart from the use in RT-PCR assays, the generated cRNA may also be suitable as a control, reference, or standard material for further RNA detection methods, including microarray analysis or linear amplification of mRNA $(9,10)$.

In conclusion, we have presented a procedure to remove template DNA from IVT-derived cRNA for use as RNA controls or RNA standards. In the presented example, the procedure yielded large amounts of virtually template DNA-free cRNA preparations, which are useful as internal control or

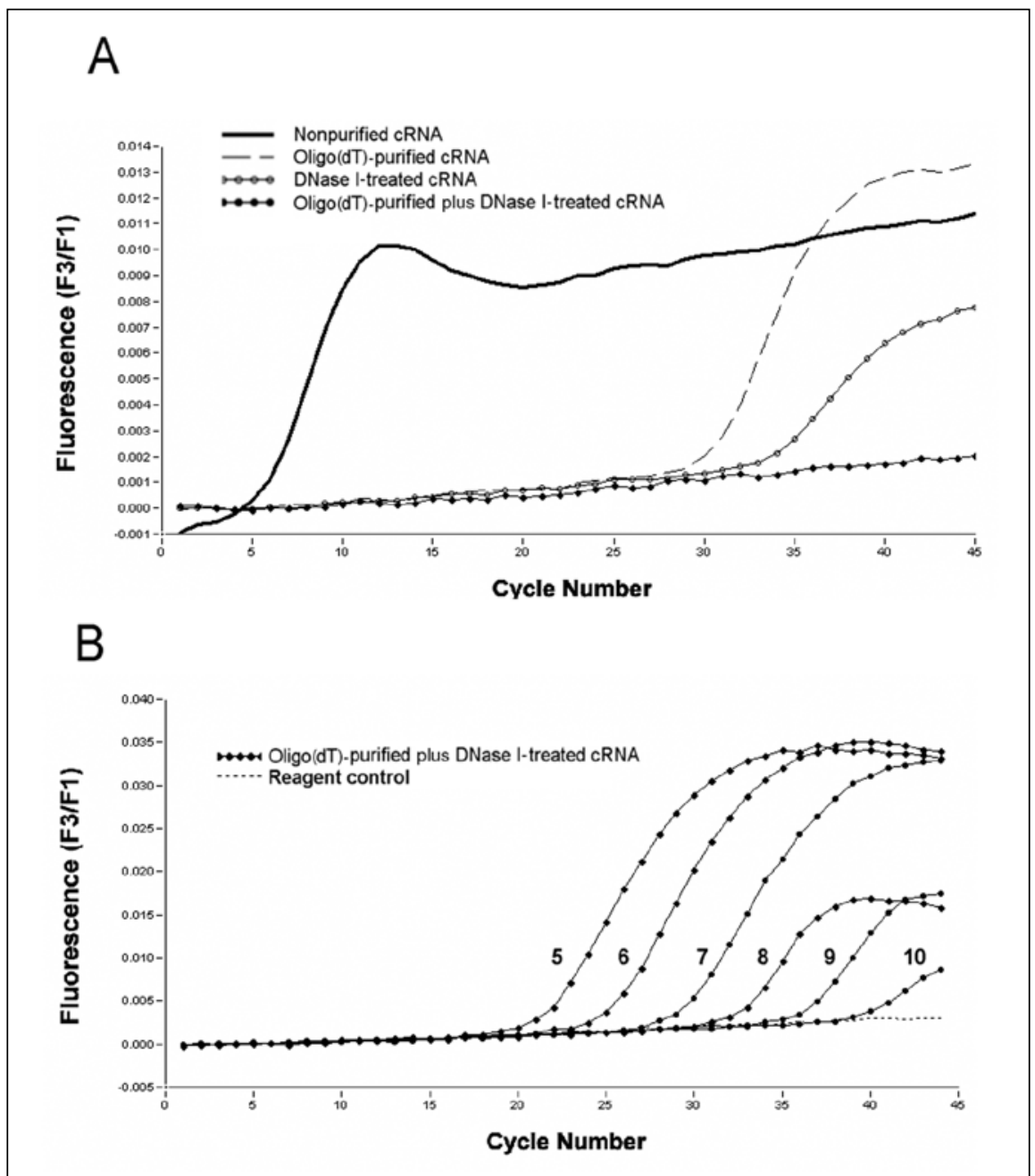

Figure 1. Analysis of purified cRNA preparations by real-time PCR with and without preceding reverse transcription. (A) Real-time PCR analysis of cRNA preparations. The cRNAs were synthesized by in vitro transcription and are either not treated (Nonpurified cRNA), subjected to oligo(dT) purification [Oligo(dT)-purified cRNA], subjected to DNase I treatment (DNase I-treated cRNA), or subjected to a combined oligo(dT) and DNase I treatment [Oligo(dT)-purified plus DNase I-purified cRNA]. Thereafter, the cRNA preparations were analyzed by real-time PCR as described in the text. (B) Reverse transcription and real-time PCR analysis of oligo(dT)-purified and DNase I-treated cRNA. The respective cRNA [Oligo(dT)-purified plus DNase I-treated cRNA] was diluted from 1:10 $10^{5}$ to $1: 10^{10}$ (numbers $5-10)$ in $\log _{10}$-fold steps with nuclease-free grade water and analyzed by reverse transcription followed by real-time PCR as described in the text. One of the two best results is presented. Reagent control refers to a sample that contained water instead of sample cRNA. Fluorescence (F3/F1) was assessed by using the LightCycler software 3.5.3. cRNA, complementary RNA.

quantification standards in an enterovirus-specific RT-PCR assay.

\section{ACKNOWLEDGMENTS}

We would like to thank Dr. Herbert Stekel, M.D., Chief, Institute of Laboratory Medicine, for his continuous support.

\section{REFERENCES}

1. Bustin, S.A. 2002. Quantification of mRNA using real-time reverse transcription PCR (RTPCR): trends and problems. J. Mol. Endocrinol. 29:23-39.

2. Evans, C.W., D.A. Wilson, and G.N. Mills. 2001. Quantitative competitive (qc) RT-PCR as a tool in biomarker analysis. Biomarkers 6:7-14.

3. Matthews, J.L.K., M. Chung, and R. Matyas. 2002. Persistent DNA contamination in competitive RT-PCR using cRNA internal control standards: identity, quantity, and control. BioTechniques 32:1412-1417.

4. Siebert, P.D. and J.W. Larrick. 1993. PCR MIMICS: competitive DNA fragments for use as internal standards in quantitative PCR. BioTechniques 14:244-249.

5. Stöcher, M., V. Leb, and J. Berg. 2003. A convenient approach to the generation of multiple internal control DNA for a panel of competitive real-time PCR assays. J. Virol. Methods 108:1-8.

6. Verstrepen, W.A., S. Kuhn, M.M. Kockx, M.E. Van de Vyvere, and A.H. Mertens. 2001. Rapid detection of enterovirus RNA in cerebrospinal fluid specimens with a novel single-tube real-time reverse transcription-PCR assay. J. Clin. Microbiol. 39:4093-4096.

7. Morrison, T.B., J.J. Weis, and C.T. Wittwer. 1998. Quantification of low copy transcripts by continuous SYBR Green I monitoring during amplification. BioTechniques 24:954-962.

8. Stöcher, M., V. Leb, M. Bozic, H.H. Kessler, G. Halwachs-Baumann, O. Landt, H. Stekel, and J. Berg. 2003. Parallel detection of five human herpes virus DNAs by a set of real-time polymerase chain reactions in a single run. J. Clin. Virol. 26:85-93.

9. Bustin, S.A. and S. Dorudi. 2002. The value of microarray techniques for quantitative gene profiling in molecular diagnostics. Trends Mol. Med. 8:269-272.

10. Sterrenburg, E., R. Turk, J.M. Boer, G.B. van Ommen, and J.T. den Dunnen. 2002. A common reference for cDNA microarray hybridisations. Nucleic Acid Res. 30:e116.

Received 24 November 2003; accepted 9 January 2004.

Address correspondence to Jörg Berg, Institute of Laboratory Medicine, General Hospital Linz, Krankenhausstrasse 9, A-4020 Linz, Austria.e-mail:joerg.berg@akh.linz.at 